Directed Search and the Bertrand Paradox

Athanasios Geromichalos

UC Davis

September 25, 2012

Paper \# 12-21

I study a directed search model of oligopolistic competition, extended to incorporate general capacity constraints, congestion effects, and pricing based on ex-post realized demand. I show that as long as any one of these ingredients is present, the Bertrand paradox will fail to hold. Hence, I argue that, despite the emphasis that has been placed by the literature on sellers' capacity constraints as a resolution to the paradox, the existence of such constraints is only a subcase of a general class of environments where the paradox fails. More precisely, Bertrand's paradox will not arise whenever the buyers' expected utility from visiting a specific seller is decreasing in that seller's realized demand.

Department of Economics

One Shields Avenue

Davis, CA 95616

(530)752-0741

http://www.econ.ucdavis.edu/working_search.cfm 


\title{
Directed Search and the Bertrand Paradox*
}

\author{
Athanasios Geromichalos ${ }^{\dagger}$ \\ Department of Economics \\ University of California, Davis
}

August 2012

\begin{abstract}
I study a directed search model of oligopolistic competition, extended to incorporate general capacity constraints, congestion effects, and pricing based on ex-post realized demand. I show that as long as any one of these ingredients is present, the Bertrand paradox will fail to hold. Hence, I argue that, despite the emphasis that has been placed by the literature on sellers' capacity constraints as a resolution to the paradox, the existence of such constraints is only a subcase of a general class of environments where the paradox fails. More precisely, Bertrand's paradox will not arise whenever the buyers' expected utility from visiting a specific seller is decreasing in that seller's realized demand.
\end{abstract}

JEL Classification: C78; D43; L13

Keywords: Directed Search, Bertrand Paradox, Capacity Constraints, Congestion Effects, State-contingent Pricing

* I am grateful to Giacomo Bonanno for his guidance and assistance. I would also like to thank Jan Eeckhout, Manolis Galenianos, Philipp Kircher, Demian Pouzo, Martine Quinzii, Dave Rapson, Kevin Salyer, and Joaquim Silvestre for useful comments and suggestions. All errors are solely my responsibility.

${ }^{\dagger}$ Contact: ageromich@ucdavis.edu 


\section{Introduction}

In economics and industrial organization (IO), the Bertrand paradox describes a situation in which the competing firms in a duopoly reach a Nash equilibrium where their price equals marginal cost. This result is characterized as a paradox because, typically, one would expect the price to equal marginal cost only in markets with a large number of firms. One resolution to the paradox, that has been emphasized by the theoretical IO literature, is the existence of constraints in the firms' capacity: if a firm sets its price at marginal cost and cannot meet the market demand, the rival firm has an incentive to deviate to a higher price, since it will still be able to attract some customers. In this paper, I study a model of oligopolistic competition in a market characterized by search frictions. I show that, despite the emphasis that has been placed by the literature on the capacity constraint resolution, the existence of such constraints is only a subcase of a more general market description where the Bertrand paradox fails to hold. In particular, I prove that Bertrand's paradox is resolved so long as the customers' expected utility from visiting a certain store is a decreasing function of the realized demand at that store.

I arrive at this generalization by building on a model that provides micro-foundations on the demand side of the market, namely the directed search model. In this model, strategic buyers, who want to consume one unit of an indivisible good, observe the prices of all sellers and visit the seller who promises them the highest expected utility. Search frictions are captured by the fact that buyers cannot coordinate their visiting strategies, and it is costly to visit more than one seller. I augment the baseline model with three ingredients: generalized capacity constraints, congestion effects, and pricing that is contingent on ex post realized demand. ${ }^{1}$ I show that, as long as any one of these three ingredients is present in the model, the Bertrand paradox ceases to exist, and this is true because the different ingredients share a common feature: they all lead to setups in which customers tend to dislike crowded stores. As a result, buyers do not necessarily visit the seller with the lowest price, which, in turn, relaxes the price war that typically leads to the Bertrand outcome.

The result described above offers a new way of looking at the role of capacity constraints in the resolution of Bertrand's paradox. With capacity constraints, buyers dislike crowded stores because these stores are associated with a higher chance of getting rationed. But other things might be going on. Perhaps sellers can serve all the visiting customers, but the buyers' valuation of the good diminishes when the store is crowded. Alternatively, if sellers can price based on ex post realized demand, they might charge more in the event that many customers show up. In all three scenarios, the paradox breaks down because buyers exert externalities on one another when visiting the same location, thus providing sellers with an incentive to charge higher prices in equilibrium. Somewhat surprisingly, this simple idea has not been formally described in the literature.

\footnotetext{
${ }^{1}$ A detailed description of these ingredients will follow. In short, the first ingredient simply means that sellers can produce up to a certain number of units of the good. The second ingredient intends to capture the fact that the consumers' valuation of the good might depend on the number of customers who get served at a certain store (e.g. people tend to dislike crowded restaurants or big lines). The third ingredient allows sellers to charge prices that depend on how many buyers show up at their store.
} 
In the baseline directed search model, $m$ sellers, usually assumed to possess only one unit of an indivisible good, post prices in order to attract some of the $n$ buyers in the market, and buyers get to visit exactly one seller. The first way in which I depart from the standard model, is by assuming that all sellers can serve up to $k \leq n$ customers, at a constant marginal cost. I treat $k$ as given and describe the symmetric equilibrium price and profit for its various realizations. I show that the equilibrium price exceeds marginal cost if and only if there are some capacity constraints, i.e. $k<n$. Since $k$ captures the intensity of frictions in the market, it is interesting to study how the equilibrium profit depends on this parameter. ${ }^{2}$ An increase in $k$ implies a higher expected number of sales but, on the other hand, it decreases the local monopoly power of sellers. I show that the symmetric equilibrium price is strictly decreasing in $k$, and the profit is hump-shaped, eventually reaching zero when $k=n$.

The second way in which I depart from the baseline model, is by assuming that buyers' utility from consuming the good depends negatively on the number of customers served by a certain seller. For instance, consumers tend to dislike crowded stores, restaurants, or amusement parks (too little space or too long waiting lines). In many cases, this type of externality might be more relevant than capacity constraints. For example, companies that provide live streaming of sporting events typically do not ration any customers who want to buy their services. Nevertheless, the quality of streaming for any given customer depends crucially (and negatively) on the total number of customers served. I show that in the presence of such congestion effects, the symmetric equilibrium price will always exceed the marginal cost, even if sellers face no capacity constraints. Surprisingly, the more severe the negative externality, the higher the equilibrium price and profit.

The third ingredient that I incorporate into the standard directed search model, is the possibility of pricing based on ex post realized demand, first studied by Coles and Eeckhout (2003). An example of such pricing behavior is an auction, where the price paid by the buyer ultimately depends on the total number of participants (and their valuation of the good). ${ }^{3}$ Assuming that sellers can price based on ex post realized demand, and that they do not face capacity constraints, I show that there exists a continuum of equilibrium prices that exceed marginal cost. This indeterminacy of equilibrium has also been documented by Coles and Eeckhout (2003). In their model, each seller has only one unit of the good available. Here, I highlight that this important result also holds true in environments where sellers' capacity is unconstrained.

The discussion above reveals that the three ingredients introduced in the model serve as collusion devices that weaken competition among sellers and help them boost equilib-

\footnotetext{
${ }^{2}$ As mentioned earlier, the search frictions in the directed search model are captured by the fact that buyers cannot coordinate their visiting strategies, and it is costly to visit more than one sellers. Hence, the element of frictions is present even if $k=n$. The case in which $k<n$ is particularly interesting because then, some sellers will be visited by more buyers than they can accommodate, while others might have idle capacity. In this case, the definition of the term "frictions" is close in spirit to Lagos (2000), who defines frictions as the coexistence of excess supply and excess demand within the same market.

${ }^{3}$ A more recent example of a company that follows pricing based on ex post realized demand is Groupon. This web-based company offers "deals of the day", but the deals are valid only if a certain number of customers participate.
} 
rium profits. Hence, sellers might have an incentive to create conditions in the market that resemble these ingredients, especially when doing so does not violate any anti-trust laws. My model has some interesting policy implications, since the welfare properties of equilibrium, under the three ingredients, are significantly different. Pricing based on ex post demand can generate high profits, but does not affect the market surplus. In this case, authorities should intervene only if they judge that the sharing rule of the surplus is unfair. On the other hand, if sellers could "fix capacities" or artificially generate congestion effects at their stores, the total surplus in the market would be reduced, but sellers would pursue these actions, since they would both lead to higher equilibrium profits.

Conceptually, the present paper is closely related to Lester (2011), who also revisits a traditional question in economics through the lens of a directed search model. In that paper, the author studies the relationship between the price setting behavior of sellers and the extent to which consumers can observe these prices before visiting a seller. He shows that the conventional wisdom, according to which in a market with more informed buyers the equilibrium price will be lower, does not necessarily hold in his model. The channel through which this important result emerges, is one that plays a crucial role in my analysis too: in the directed search model, due to capacity constraints, buyers do not necessarily visit the seller with the lowest price.

The fact that Bertrand's paradox will fail to hold in a directed search model, where sellers' capacity is constrained, is well established in the literature (for instance, see Peters (1984)). Here, I argue that the paradox will not arise in any environment where buyers tend to dislike crowded stores, and that the existence of capacity constraints is just one example of such environments. Most papers in the directed search literature make an extreme, but convenient, assumption on sellers' capacity constraints: they simply assume that sellers possess one unit of the good. Some exceptions are Lester (2010), Tan (2010), and Watanabe (2010). The idea that buyers' valuation of the good depends on the number of customers who get served at a certain store is also exploited in Geromichalos (2009). There is also a number of papers which study pricing based on ex post realized demand, a la Coles and Eeckhout (2003). Examples of such papers include Julien, Kennes, and King (2000), Virag (2007), and Jacquet and Tan (2012).

Finally, this paper is related to a number of papers, in the theoretical IO literature, which have proposed various resolutions to Bertrand's paradox. ${ }^{4}$ One strand of the literature focuses on product differentiation (for example, see Shaked and Sutton (1982)). Another strand suggests that the Bertrand paradox breaks down if sellers interact repeatedly (for example, see Dudey (1992)). In static environments with a homogeneous good, the resolution that has attracted the greatest share of attention is the existence of capacity constraints. This idea dates back to Edgeworth (1897). In other notable work, Kreps and Scheinkman (1983) consider a game where sellers choose their capacity and their prices sequentially, and show that the resulting equilibrium coincides with Cournot's (Cournot (1838)) outcome. The present paper attempts to offer a new perspective, by arguing that the existence of capacity constraints is just a special case of a general class of environments where the paradox fails to hold.

\footnotetext{
${ }^{4} \mathrm{I}$ do not attempt to provide a complete survey of this literature here.
} 
The rest of this paper is organized as follows. In Section 2, I present a directed search model, extended to incorporate general capacity constraints, congestion effects, and pricing based on ex post realized demand. Sections 3, 4, and 5 describe equilibrium in environments containing the ingredients described above, in isolation. In Section 6, I compare the welfare properties of equilibrium in the three environments and discuss the model's policy implications. Section 7 concludes.

\section{The Model}

In this section, in the interest of generality, I describe a model of price posting and consumer search with the following three ingredients: 1) sellers face capacity constraints, 2) buyers' valuation of the good depends negatively on the total number of customers who get served at a certain store, and 3) sellers are able to post prices that depend on ex post realized demand. In the sections that follow, these three ingredients are not present simultaneously. This is intentional. The purpose is to highlight that only one of them is necessary in order to generate equilibria where the Bertrand Paradox fails to hold.

My model builds on Burdett, Shi, and Wright (2001). I consider a market with $n$ buyers and $m$ sellers, $n, m \geq 2$ and finite. All agents are risk neutral. All buyers are identical and anonymous, and each wishes to purchase one unit of an indivisible good. Each seller can produce $i \leq k$ units of the good at a linear cost $c(i)=c i, c>0$. Hence, $k$ is the capacity constraint that sellers face. I treat this parameter as given and describe the pricing decisions of sellers based on the various realizations of $k$. As it is common in the directed search literature, I assume that that buyers can only visit one seller, and they cannot coordinate their visiting strategies. It is the combination of these two assumptions that captures the notion of search frictions in my model.

Buyers' utility from consuming the good depends on the number of customers who get served at a certain location: if a seller serves $i \leq n$ customers, the utility enjoyed by each customer is $u(i)$, and for any $h<i, u(h) \geq u(i)$. This assumption aims to capture the existence of negative consumption externalities (people dislike to eat in over-crowded restaurants), or congestion effects, such as lines or long waiting times. Furthermore, define $\sigma(i) \equiv i[u(i)-c]$, i.e. the net surplus generated by a seller who serves $i$ customers. I assume that $\sigma(i)$ is non-decreasing for all $i \leq k$. This assumption guarantees that it is optimal for sellers to serve as many buyers as their capacity allows. Hence, rationing will occur only if the number of visiting buyers exceeds $k$.

The exchange process consists of two stages. At the first stage, given the value of $k$ (which is common for all sellers), each seller posts a price advertisement, taking as given the strategies of her $m-1$ competitors. A price announcement for seller $j$ is a vector

$\mathbf{p}^{j}=\left(p_{1}^{j}, \ldots, p_{n}^{j}\right)$, where $p_{i}^{j}$ is the price paid to seller $j$ by customers who get served if that seller gets visited by $i$ buyers. At the second stage, buyers observe all the advertisements and choose a probability of visiting each seller, taking as given the strategies of other buyers. Let $i$ represent the number of buyers who show up at seller $j$. If $i \leq k$, all buyers get served, but if $i>k$, the seller serves exactly $k$ buyers chosen at random. The buyers 
who get served enjoy a net utility of $u(\min \{k, i\})-p_{i}^{j}$, and those who get rationed (if any) get a payoff of zero. I assume that sellers commit to their advertisements.

In order to have all agents participate in the trading process, the expected utility and profit generated by the posted pricing schemes have to be non-negative. Moreover, I assume that buyers can walk away from the trading process at any time and obtain utility equal to zero. Hence, I require that in every contingency, the price paid by buyers who get served cannot exceed their valuation of the good, i.e. for all $i \leq n, p_{i}^{j} \leq$ $u(\min \{k, i\}) .^{5}$ I refer to these inequalities as ex post participation constraints. I do not impose any assumptions that prevent prices from being smaller than the marginal cost, or even negative, in some states, provided that they lead to a non-negative expected profit.

As it is common in the directed search literature, I focus on symmetric equilibria in which buyers play mixed strategies in the subgame. A rich set of equilibria in pure strategies exist, but they are considered implausible since they require an unreasonable degree of coordination among the buyers, in the sense that a buyer needs to know where other buyers are going. ${ }^{6}$ Equilibria in mixed strategies have gained popularity in the literature, precisely because they are consistent with the frictions that directed search models were designed to capture in the first place.

Consider ex ante payoffs. Suppose that seller $j$, who announces $\mathbf{p}^{j}$, gets visited by an arbitrary buyer with probability $\theta$. The expected utility of a buyer who visits seller $j$ is

$$
U^{j}\left(\mathbf{p}^{j}, \theta\right)=\sum_{i=1}^{n}\left(\begin{array}{c}
n-1 \\
i-1
\end{array}\right)(1-\theta)^{n-i} \theta^{i-1} \frac{\min \{i, k\}}{i}\left[u(\min \{i, k\})-p_{i}^{j}\right],
$$

where $\left(\begin{array}{c}n-1 \\ i-1\end{array}\right)$ denotes the binomial coefficient, and the expected profit of seller $j$ is

$$
\pi^{j}\left(\mathbf{p}^{j}, \theta\right)=\sum_{i=1}^{n}\left(\begin{array}{l}
n \\
i
\end{array}\right)(1-\theta)^{n-i} \theta^{i} \min \{i, k\}\left(p_{i}^{j}-c\right) .
$$

For future reference, it is useful to define the function ${ }^{7}$

$$
H(i, n, \theta) \equiv\left(\begin{array}{c}
n-1 \\
i-1
\end{array}\right)(1-\theta)^{n-i} \theta^{i-1} \frac{1}{i} .
$$

Given the definition in $(1)$ and noticing that $\left(\begin{array}{c}n \\ i\end{array}\right)=(n / i)\left(\begin{array}{c}n-1 \\ i-1\end{array}\right)$, allows one to re-write the expected utility and profit functions above as

$$
\begin{aligned}
U^{j}\left(\mathbf{p}^{j}, \theta\right) & =\sum_{i=1}^{n} H(i, n, \theta) \min \{i, k\}\left[u(\min \{i, k\})-p_{i}^{j}\right], \\
\pi^{j}\left(\mathbf{p}^{j}, \theta\right) & =n \theta \sum_{i=1}^{n} H(i, n, \theta) \min \{i, k\}\left(p_{i}^{j}-c\right) .
\end{aligned}
$$

\footnotetext{
${ }^{5}$ For reasons that will become apparent later, in Section 4, I adopt a stronger assumption in order to guarantee existence of equilibrium for all parameter values.

${ }^{6}$ For a more detailed discussion on pure strategy equilibria see Burdett, Shi, and Wright (2001).

${ }^{7}$ The term $H(i, n, \theta)$ represents the probability with which a buyer who visits seller $j$ gets served, when seller $j$ has only one unit of the good available and a total number of $i$ customers show up.
} 
Notice that the term $\sum_{i=1}^{n} H(i, n, \theta) \min \{i, k\} p_{i}^{j}$ appears in both (2) and (3). This observation will be key for solving the sellers' profit maximization problem.

To conclude this section, I present two important algebraic facts, that I will use repeatedly in the paper. Recall the definition of $H(i, n, \theta)$ in (1) and also define the function

$$
F(i, n, \theta) \equiv \frac{i-n \theta}{1-\theta}
$$

The following relationships hold true:

$$
\begin{array}{ll}
\text { Fact 1: } & \sum_{i=1}^{n} H(i, n, \theta) i=1, \\
\text { Fact 2: } & \sum_{i=1}^{n} H(i, n, \theta) F(i, n, \theta) i=1 .
\end{array}
$$

Fact 1 is obvious. ${ }^{8}$ Fact 2 is not too hard to show with pencil and paper, but it can also be easily verified using some software such as Mathematica or Maple. Interestingly, although the functions $H, F$ look quite complex when taken individually, the sum of their product, also multiplied by $i$, turns out to be equal to the unit.

\section{Capacity Constraints}

In this section, I assume that sellers can accommodate only up to $k \leq n$ buyers, and I ignore congestion effects and sophisticated pricing mechanisms. As I mentioned above, the purpose of this strategy is to stress that only one of the three ingredients of the model described in Section 2 is necessary in order to generate equilibria where the Bertrand Paradox is resolved. More precisely, I assume that $u(i)=u>c$, for all $i \leq k .{ }^{9}$ Also, the typical seller $j$ can only advertise a unique price, $p_{i}^{j}=p^{j}$, for all $i \leq n$. Under these specifications, equations (2) and (3) become

$$
\begin{aligned}
U^{j}\left(p^{j}, \theta\right) & =\left(u-p^{j}\right) \sum_{i=1}^{n} H(i, n, \theta) \min \{i, k\} \\
\pi^{j}\left(p^{j}, \theta\right) & =n \theta\left(p^{j}-c\right) \sum_{i=1}^{n} H(i, n, \theta) \min \{i, k\} .
\end{aligned}
$$

The term $\pi^{j}\left(p^{j}, \theta\right)$ is the expected profit of seller $j$, when she announces $p^{j}$ and gets visited by an arbitrary buyer with probability $\theta$. The term $U^{j}\left(p^{j}, \theta\right)$ is the expected utility of a buyer who visits seller $j$.

\footnotetext{
${ }^{8}$ The expression $H(i, n, \theta) i$ is the probability with which a buyer's preferred seller gets visited by a total of $i$ buyers. Hence, the sum over all possible events must add up to 1.

${ }^{9}$ One of the assumptions adopted in Section 2 required the function $\sigma$ to be non-decreasing for all $i \leq k$. Here, $\sigma(i)=i(u-c)$ and, therefore, $u>c$ guarantees that $\sigma(i)$ is strictly increasing.
} 
The typical seller chooses her price, taking as given the prices of her rival sellers. Since, the goal here is to construct symmetric equilibria, I assume that all other sellers post the (same) price $\tilde{p}$. Seller $j$ sets $p^{j}$ understanding that, in the second stage, buyers will observe $p^{j}, \tilde{p}$ and will determine the probabilities with which they visit each seller, such that they are indifferent among all sellers (recall that buyers are assumed to play mixed strategies in the subgame). Hence, if $\theta$ represents the probability with which an arbitrary buyer visits seller $j$, then the probability with which that buyer visits any other seller is given by $\tilde{\theta}=\tilde{\theta}(\theta)=(1-\theta) /(m-1) .{ }^{10}$ Formally, seller $j$ solves

$$
\begin{gathered}
\max _{p^{j}} \pi^{j}\left(p^{j}, \theta\right) \\
\text { s.t. } U^{j}\left(p^{j}, \theta\right)=U^{l}(\tilde{p}, \tilde{\theta}) .
\end{gathered}
$$

The term $U^{l}(\tilde{p}, \tilde{\theta})$ is the expected utility that buyers obtain if they visit any seller $l \neq j$. It is described by (5), if one substitutes $p^{j}$ with $\tilde{p}$ and $\theta$ with $\tilde{\theta}$, i.e. the price and visiting probability associated with seller $l$.

Having established the sellers' problem, I now state the first main result of this section.

Lemma 1. In the unique symmetric equilibrium, every buyer visits each seller with probability $\theta^{*}=1 / m$, and all sellers announce the price

$$
p^{*}(n, m ; k)=\frac{\sum_{i=1}^{n} H\left(i, n, \frac{1}{m}\right) \min \{i, k\}\left\{\frac{m}{m-1}\left[1-F\left(i, n, \frac{1}{m}\right)\right] u+F\left(i, n, \frac{1}{m}\right) c\right\}}{\frac{1}{m-1} \sum_{i=1}^{n} H\left(i, n, \frac{1}{m}\right) \min \{i, k\}\left[m-F\left(i, n, \frac{1}{m}\right)\right]},
$$

where the functions $H$ and $F$ are defined in (1) and (4), respectively.

Proof. A key observation is that the term $p^{j} \sum_{i=1}^{n} H(i, n, \theta) \min \{i, k\}$ appears in both $\pi^{j}\left(p^{j}, \theta\right)$ and $U^{j}\left(p^{j}, \theta\right)$. Solving the constraint in the seller's problem with respect to this term and substituting into the profit, reveals that seller $j$ 's objective function is

$$
\max _{\theta} n \theta\left[(u-c) \sum_{i=1}^{n} H(i, n, \theta) \min \{i, k\}-U^{l}(\tilde{p}, \tilde{\theta})\right] .
$$

Hence, the seller's objective can be written as a function of the variable $\theta$ only. Seller $j$ 's best response satisfies the first-order condition

$$
\begin{gathered}
(u-c) \sum_{i=1}^{n} H(i, n, \theta) \min \{i, k\}-U^{l}(\tilde{p}, \tilde{\theta})+ \\
+\theta\left[(u-c) \sum_{i=1}^{n} \frac{\partial H(i, n, \theta)}{\partial \theta} \min \{i, k\}-(u-\tilde{p}) \sum_{i=1}^{n} \frac{\partial H(i, n, \tilde{\theta})}{\partial \tilde{\theta}} \frac{d \tilde{\theta}}{d \theta} \min \{i, k\}\right]=0 .
\end{gathered}
$$

\footnotetext{
${ }^{10}$ Notice that the analysis is consistent with the fact that the market studied here is an oligopoly. The number of sellers, $m$, is small enough so that when a seller chooses her price, she understands that this will affect not only the probability with which buyers visit her store in the second stage, but also the probability with which buyers visit her rivals. If $m$ is very large, the effect of $p^{j}$ on $\tilde{\theta}$ is so small that can be safely ignored. This is equivalent to the market utility approach often employed in the literature. Under this approach, sellers maximize profits subject to the constraint of providing visiting buyers with a certain level of utility, which they take as given. Examples of papers which build on this method are Montgomery (1991), Lang (1991), Acemoglu and Shimer (1999), and Galenianos and Kircher (2009).
} 
Notice a few important points. First, $d \tilde{\theta} / d \theta=-1 /(m-1)$. Second,

$$
\frac{\partial H(i, n, x)}{\partial x}=H(i, n, x)\left(\frac{i-1}{x}-\frac{n-i}{1-x}\right) .
$$

Third, since the focus is on symmetric equilibria, one can now (after obtaining the firstorder condition) impose symmetry: all sellers must post the same price, $p^{j}=\tilde{p}=p^{*}$, which, in turn, implies that all buyers will visit each seller with the same probability, $\theta=\tilde{\theta}=\theta^{*}=1 / m$. Once these three observations are incorporated into (8), we are left with one equation in one unknown, $p^{*}$. Solving with respect to this term, after some manipulations, yields the desired result.

Lemma 1 provides a closed form solution for the unique symmetric equilibrium price, for any $n, m, k$. Naturally, $p^{*}$ is increasing in $n$ and decreasing in $m$. More interestingly, $p^{*}$ is strictly decreasing in the capacity of sellers. Intuitively, given the frictions in the environment (inability of buyers to coordinate), a lower $k$ implies a higher probability of rationing. This provides sellers with a greater local monopoly power, and allows them to charge a higher per unit price. The monotonicity of $p^{*}$ in $k$ is an important finding, which, to my knowledge, has not been shown in any other paper in the directed search literature. ${ }^{11}$ Since the proof contains some technical details that are not essential for the understanding of the model, it is relegated to the appendix. To illustrate (7), let $n=m=2$ and $k=1$. Then, one can easily show that $p^{*}(2,2 ; 1)=0.5(u+c)$. Compare this result to the one found in Burdett, Shi, and Wright (2001). In that paper, sellers have one unit of the good $(k=1)$, and the parameters $u, c$ are normalized to 1 and 0 , respectively. For $n=m=2$, the authors find $p^{*}=0.5$. Thus, the two results coincide.

I now focus on the equilibrium profits and state the main result of this section. From (6), in the symmetric equilibrium, each seller has an expected profit equal to

$$
\pi^{*}(n, m ; k)=\frac{n}{m}\left[p^{*}(n, m ; k)-c\right] \sum_{i=1}^{n} H\left(i, n, \frac{1}{m}\right) \min \{i, k\} .
$$

Since every term inside the summation is positive, sellers make a positive profit if and only if $p^{*}$ is greater than the marginal cost. The next proposition establishes that this is indeed the case as long as sellers face some capacity constraints.

Proposition 1. a) If $k=n$, then $p^{*}(n, m ; k)=c$ and, hence, $\pi^{*}(n, m ; k)=0$.

b) If $k<n$, then $p^{*}(n, m ; k)>c$ and, hence, $\pi^{*}(n, m ; k)>0$.

Proof. a) For the economy of space, I write $H(i)$ for $H(i, n, 1 / m)$ and $F(i)$ for $F(i, n, 1 / m)$. First, notice that if $k=n$, one can write

$$
p^{*}(n, m ; k)=\frac{\sum_{i=1}^{n} H(i) i\left\{\frac{m}{m-1}[1-F(i)] u+F(i) c\right\}}{\frac{1}{m-1} \sum_{i=1}^{n} H(i) i[m-F(i)]}=
$$

${ }^{11}$ As mentioned in the Introduction, most papers in the directed search literature assume that $k$ is fixed, and usually equal to one. 


$$
=\frac{\frac{m}{m-1} u \sum_{i=1}^{n} H(i) i[1-F(i)]+c \sum_{i=1}^{n} H(i) i F(i)}{\frac{1}{m-1}\left[m \sum_{i=1}^{n} H(i) i-\sum_{i=1}^{n} H(i) F(i) i\right]}=c,
$$

where the last equality follows directly from Facts 1 and 2 . Thus, if $k=n$, the equilibrium profit for sellers is zero, and the Bertrand result emerges.

b) To see why any $k<n$ is sufficient for $p^{*}(n, m ; k)>c$, recall that $p^{*}(n, m ; k)$ is strictly decreasing in $k$, for all $k=1, \ldots, n$ (see proof in the appendix). In other words, for all $k=1, \ldots, n-1, p^{*}(n, m ; k)>p^{*}(n, m ; k+1)$. Since $p^{*}(n, m ; n)=c$, we have that $p^{*}(n, m ; k)>c$ for all $k<n$.

As long as sellers face some capacity constraints $(k<n)$, they achieve strictly positive profit in the symmetric equilibrium. The equilibrium profit is the product of the markup term $p^{*}(n, m ; k)-c$ and the expected number of sales. ${ }^{12}$ Clearly, expected sales increase in $k$. However, these increases get very small as $k$ gets close to $n$. On the other hand, $p^{*}(n, m ; k)$ is decreasing in $k$. Typically, $\pi^{*}(n, m ; k)$ is increasing for small values of $k$, and eventually it decreases until $\pi^{*}(n, m ; n)=0$. One exception to this rule occurs when the market tightness $n / m$ is very small. In this case, the increase in expected sales, following a rise of $k$ from 1 to 2 , is already very small. Thus, the negative effect of increasing $k$ (lower price) is dominant even for very small values of $k$, and the symmetric equilibrium profit is maximized for $k=1$. For example, if $n=5, m=3$, $\pi^{*}(5,3 ; 1)=0.493>0.438=\pi^{*}(5,3 ; 2)$.

\section{Consumption Externalities}

In this section, I assume that sellers can accommodate all the buyers who visit their store. Also, like in the previous section, seller $j$ can post a unique price, $p_{i}^{j}=p^{j}$, for all $i \leq n$. The interesting feature of this section is that buyers' valuation of the good depends on the number of customers who get served at their preferred location. If the seller serves $i$ customers, the utility enjoyed by each is given by $u(i)$, and I assume that for any $h<i, u(h) \geq u(i)$. Hence, this environment is characterized by negative consumption externalities or, alternatively, congestion effects.

In this section (only), I relax the ex post participation assumption, that is, I do not assume that the posted price should be no greater than the buyer's valuation in every single contingency. The reason is straightforward. Here, $k=n, u(i)$ is non-increasing in $i$, and sellers can only post one price. If $n$ is large and $u(n)$ is strictly decreasing (say $u(i)=1 / i)$, there will always exist events in which the buyer's valuation for the good is tiny. Hence, it might be impossible to find a single equilibrium price, $p^{*}$, that satisfies $p^{*} \leq u(i)$ for very large $i$ 's. To guarantee existence of equilibrium, I will only require that the posted prices lead to a non-negative expected utility for the buyers. In some sense,

\footnotetext{
12 This follows from $(9):(n / m) H(i, n, 1 / m)$ is the probability of getting $i$ customers. These visits will translate into sales as long as $i \leq k$. Hence, the number of expected sales is $(n / m) H(i, n, 1 / m) \min \{i, k\}$.
} 
this is equivalent to assuming that the buyer commits to paying the posted price at the location she picked. Notice that doing so is in fact optimal, if the buyer is assumed to not know the total number of visiting customers when she makes the payment. ${ }^{13}$

Under these specifications, and using Fact 1, one can re-write equations (2),(3) as ${ }^{14}$

$$
\begin{aligned}
U^{j}\left(p^{j}, \theta\right) & =\sum_{i=1}^{n} H(i, n, \theta) i u(i)-p^{j}, \\
\pi^{j}\left(p^{j}, \theta\right) & =n \theta\left(p^{j}-c\right) .
\end{aligned}
$$

The term $\pi^{j}\left(p^{j}, \theta\right)$ is the expected profit of seller $j$, when she announces $p^{j}$ and gets visited by an arbitrary buyer with probability $\theta$. The term $U^{j}\left(p^{j}, \theta\right)$ is the expected utility of a buyer who visits seller $j$. As in the previous section, suppose that all sellers but $j$ post $\tilde{p}$, and let $\tilde{\theta}=\tilde{\theta}(\theta)=(1-\theta) /(m-1)$ denote the probability with which the arbitrary buyer visits each of these sellers. Then, seller $j$ solves

$$
\begin{gathered}
\max _{p^{j}} \pi^{j}\left(p^{j}, \theta\right) \\
\text { s.t. } U^{j}\left(p^{j}, \theta\right)=U^{l}(\tilde{p}, \tilde{\theta}) .
\end{gathered}
$$

In words, seller $j$ chooses $p^{j}$ taking $\tilde{p}$ as given and realizing that, in the second stage, $\theta$ and $\tilde{\theta}$ will adjust so that buyers are indifferent between visiting seller $j$ or any other seller $l$. The next lemma describes the equilibrium prices.

Lemma 2. In the unique symmetric equilibrium, every buyer visits each seller with probability $\theta^{*}=1 / m$, and all sellers announce the price

$$
p^{*}(n, m)=c+\frac{m}{m-1} \sum_{i=1}^{n} H\left(i, n, \frac{1}{m}\right) i\left[1-F\left(i, n, \frac{1}{m}\right)\right] u(i),
$$

where the functions $H$ and $F$ are defined in (1) and (4), respectively.

Proof. The proof follows the same steps as that of Lemma 1. One can replace $p^{j}$ from the constraint of the seller's problem into the profit function, and re-write seller $j$ 's objective only as a function of the probability $\theta$,

$$
\max _{\theta} n \theta\left\{\sum_{i=1}^{n}[H(i, n, \theta)-H(i, n, \tilde{\theta})] i u(i)+\tilde{p}-c\right\} .
$$

\footnotetext{
${ }^{13}$ Relaxing the ex post participation assumption guarantees existence of a symmetric equilibrium, but it does not affect the properties of this equilibrium. Put differently, if this assumption is maintained, existence of equilibrium is not guaranteed for all parameter values. However, when equilibrium does exist, it still satisfies the property highlighted in the forthcoming Proposition 2, i.e. $p^{*}>c$. Hence, relaxing the ex post participation requirement does not change the nature of the main result.

14 Since here $k=n$, the buyer pays $p^{j}$ with certainty, and her net expected utility depends on the total number of buyers who visit seller $j$. The seller's profit is equal to the number of expected sales, $n \theta$, times the per unit mark-up $p^{j}-c$.
} 
Taking the first-order condition with respect to $\theta$, imposing the usual conditions of symmetry, and solving with respect to the price yields

$$
p^{*}(n, m)=\frac{\sum_{i=1}^{n} H\left(i, n, \frac{1}{m}\right) i\left\{\frac{m}{m-1}\left[1-F\left(i, n, \frac{1}{m}\right)\right] u(i)+F\left(i, n, \frac{1}{m}\right) c\right\}}{\frac{1}{m-1} \sum_{i=1}^{n} H\left(i, n, \frac{1}{m}\right) i\left[m-F\left(i, n, \frac{1}{m}\right)\right]} .
$$

The formula reported in (12) follows from using Facts 1 and 2 in the expression above.

Lemma 2 provides a closed form solution for the equilibrium price, for any $n, m$, and for any function $u(i)$. As an example, suppose that $n=m=2$. Since $k=n$, we know from the analysis of Section 3 that, if $u(i)=u$ for all $i$, then $p^{*}(2,2 ; 2)=c$. But now suppose that if both buyers show up at the same seller, their utility from consuming the good is cut down by half, i.e. $u(1)=u$ and $u(2)=u / 2$. Under this specification, it is easy to verify that $p^{*}=c+u / 2$, and the equilibrium price exceeds the marginal cost. In what follows I generalize this result for any parameter values. Notice that, from (11), the symmetric equilibrium profit, $\pi^{*}(n, m)$, will be positive if and only if $p^{*}>c$.

Proposition 2. a) If $u(i)=u$ for all $i$, then $p^{*}(n, m)=c$ and, therefore, $\pi^{*}(n, m)=0$.

b) If for all $i \in\{1, \ldots, n-1\}, u(i) \geq u(i+1)$, with strict inequality for some $i$, then $p^{*}(n, m)>c$ and, therefore, $\pi^{*}(n, m)>0$.

Proof. It is understood that $H(i)=H(i, n, 1 / m)$ and $F(i)=F(i, n, 1 / m)$.

a) If $u(i)=u$, one can write

$$
p^{*}(n, m)=c+\frac{m}{m-1} u \sum_{i=1}^{n} H(i) i[1-F(i)]=c,
$$

since, from Facts 1 and $2, \sum_{i=1}^{n} H(i) i[1-F(i)]=0$.

b) If $u(i) \neq u, p^{*}(n, m)$ is given by (12). As pointed out earlier, $\sum_{i=1}^{n} H(i) i[1-F(i)]=$ 0 . Moreover, the function $1-F(i)$ is strictly decreasing in $i$, for all $i \leq n$, and satisfies $1-F(1)=(n-1) /(m-1)>0$, and $1-F(n)=1-n<0$. Therefore, as long as $u(i)$ is decreasing (in the precise sense that $u(i) \geq u(i+1)$, with strict inequality for some $i$ ), multiplying the terms $H(i) i[1-F(i)]$ with $u(i), i=1, \ldots, n$, assigns greater weights on the relatively greater values. Since $\sum_{i=1}^{n} H(i) i[1-F(i)]=0$, it must be that $\sum_{i=1}^{n} H(i) i[1-F(i)] u(i)>0$. Then, from $(12), p^{*}(n, m)>c$ follows immediately.

Proposition 2 indicates that when the buyers' valuation of the good depends on the total number of customers who visit a certain location, sellers can achieve positive profits in the symmetric equilibrium, even in the absence of any capacity constraints. To my knowledge, this result has not been documented in the directed search (or any other) literature. It highlights that sellers can use the state dependent valuation of the good as a collusion device in order to increase equilibrium profits. In Section 6, I argue that this result has some important policy implications, since it indicates that sellers have an incentive to make the buyers' valuation dependent on ex post realized demand artificially 
(for example, by allowing big lines at their stores).

I now illustrate Proposition 2 using a concrete example. Let $u(i)=v-\kappa(i-1)$, with $\kappa>0$ and $v>c$. Hence, the buyer's valuation decreases linearly in the number of other buyers that visit the same store. To guarantee that $\sigma(i)=i[v-c-\kappa(i-1)]$ is non-decreasing, I impose the restriction $\kappa \leq(v-c) /(2 n-1)$. Under this specification, the equilibrium price becomes

$$
p^{*}(n, m)=c+\kappa \frac{m}{m-1} \sum_{i=1}^{n} H\left(i, n, \frac{1}{m}\right)\left[F\left(i, n, \frac{1}{m}\right)-1\right] i^{2},
$$

and one can show that $\sum_{i=1}^{n} H(i, n, 1 / m)[F(i, n, 1 / m)-1] i^{2}=(n-1) / m$, implying that

$$
p^{*}(n, m)=c+\kappa \frac{n-1}{m-1} .
$$

Clearly, the equilibrium price and the profit, $\pi^{*}=\kappa[n(n-1)][m(m-1)]^{-1}$, are increasing in the parameter $\kappa$, which captures the reduction in the valuation of the good when one additional buyer visits a certain store. When $\kappa$ is large, the expected utility from visiting a store with many customers is diminished. Thus, buyers are willing to choose a seller with a higher price, hoping that this seller will be visited by fewer customers. This, in turn, gives an incentive to sellers to post higher prices. Of course, in symmetric equilibrium, all sellers get the same number of expected buyers. However, the existence of congestion effects serves as a collusion device allowing sellers to achieve positive profit, even if $k=n$.

\section{$5 \quad$ Pricing Based on Ex Post Realized Demand}

In this section, I maintain the assumption $k=n$. Also, like in Section 3, I assume that $u(i)=u>c$, for all $i \leq n$. The interesting results of this section are driven by the fact that sellers can post prices that are contingent on ex post realized demand. More precisely, the typical seller $j$ can advertise a vector $\mathbf{p}^{j}=\left(p_{1}^{j}, \ldots, p_{n}^{j}\right)$, where $p_{i}^{j}$ is the price paid to seller $j$ by all customers when $i$ of them show up. Ex post participation of the buyers requires that, for all $i \leq n, p_{i}^{j} \leq u$. Prices in some states can be smaller than the marginal cost, or even negative (and sellers are committed to honor these announcements), as long as they lead to a non-negative expected profit.

Under these specifications, equations (2) and (3) become ${ }^{15}$

$$
\begin{aligned}
U^{j}\left(\mathbf{p}^{j}, \theta\right) & =u-\sum_{i=1}^{n} H(i, n, \theta) i p_{i}^{j}, \\
\pi^{j}\left(\mathbf{p}^{j}, \theta\right) & =n \theta\left[\sum_{i=1}^{n} H(i, n, \theta) i p_{i}^{j}-c\right] .
\end{aligned}
$$

\footnotetext{
${ }^{15}$ In this environment, buyers enjoy utility $u$ with certainty, and they pay a price that depends on the total number of customers who visit seller $j$. This is in contrast with Section 4 (equation (10)), where buyers pay a certain price, and the valuation of the good depends on ex post realized demand.
} 
The term $\pi^{j}\left(\mathbf{p}^{j}, \theta\right)$ is the expected profit of seller $j$, when she posts the price vector $\mathbf{p}^{j}$ and gets visited by an arbitrary buyer with probability $\theta$. The term $U^{j}\left(\mathbf{p}^{j}, \theta\right)$ is the expected utility of a buyer who visits seller $j$. As in the previous sections, suppose that all sellers but $j$ post $\tilde{\mathbf{p}}=\left(\tilde{p}_{1}, \ldots, \tilde{p}_{n}\right)$, and let $\tilde{\theta}=\tilde{\theta}(\theta)=(1-\theta) /(m-1)$ denote the probability with which the arbitrary buyer visits each of these sellers. Seller $j$ solves

$$
\begin{gathered}
\max _{\mathbf{p}^{j}} \pi^{j}\left(\mathbf{p}^{j}, \theta\right) \\
\text { s.t. } U^{j}\left(\mathbf{p}^{j}, \theta\right)=U^{l}(\tilde{\mathbf{p}}, \tilde{\theta}) .
\end{gathered}
$$

In words, seller $j$ advertises a pricing schedule $\mathbf{p}^{j}$ taking $\tilde{\mathbf{p}}$ as given and realizing that, in the second stage, $\theta$ and $\tilde{\theta}$ will adjust so that buyers are indifferent between visiting seller $j$ or any other seller $l$. The next lemma describes equilibrium prices.

Lemma 3. Every price vector $\boldsymbol{p}^{*}=\left(p_{1}^{*}, \ldots, p_{n}^{*}\right)$ that satisfies

$$
\begin{gathered}
\frac{1}{m-1} \sum_{i=1}^{n} H\left(i, n, \frac{1}{m}\right) i\left[m-F\left(i, n, \frac{1}{m}\right)\right] p_{i}^{*}=c, \\
\frac{n}{m}\left[\sum_{i=1}^{n} H\left(i, n, \frac{1}{m}\right) i p_{i}^{*}-c\right] \geq 0
\end{gathered}
$$

and $p_{i}^{*} \leq u$, for all $i=\{1, \ldots, n\}$, together with a strategy for the buyers to visit each seller with probability $\theta^{*}=1 / m$, constitutes a symmetric equilibrium.

Proof. The proof follows the same steps as those of Lemmas 1 and 2. One can replace the term $\sum_{i=1}^{n} H(i, n, \theta) i p_{i}^{j}$ from the constraint of the seller's problem into the profit function, and re-write seller $j$ 's objective only as a function of $\theta$,

$$
\max _{\theta} n \theta\left[\sum_{i=1}^{n} H(i, n, \tilde{\theta}) i \tilde{p}_{i}-c\right] \text {. }
$$

Taking the first-order condition in (15) with respect to $\theta$ and imposing the usual conditions of symmetry yields (13). The left-hand side in (14) is the equilibrium expected profit. Hence, (14) guarantees that expected profit is non-negative. Expected net utility is guaranteed to be non-negative by the ex post participation constraints.

Lemma 3 reveals that there are $n-1$ degrees of freedom in determining the equilibrium objects $p_{1}^{*}, \ldots, p_{n}^{*}$. Hence, equilibrium prices are not uniquely pinned down. This result is not unique to my model. It was first established by Coles and Eeckhout (2003). However, in that paper, sellers have only one unit of the good available. This paper demonstrates that the well-known indeterminacy result, documented by Coles and Eeckhout (2003), also holds true in environments without capacity constraints. To my knowledge, this important extension has not been pointed out in the literature. I will return to explain the intuition behind this finding after describing equilibrium profits. 
Let $\pi^{*}\left(n, m ; \mathbf{p}^{*}\right)$ denote the symmetric equilibrium expected profit if price $\mathbf{p}^{*}$ prevails, and let $U^{*}\left(n, m ; \mathbf{p}^{*}\right)$ be the analogue expression for equilibrium net expected utility. Thus,

$$
\begin{aligned}
\pi^{*}\left(n, m ; \mathbf{p}^{*}\right) & =\frac{n}{m}\left[\sum_{i=1}^{n} H\left(i, n, \frac{1}{m}\right) i p_{i}^{*}-c\right], \\
U^{*}\left(n, m ; \mathbf{p}^{*}\right) & =u-\sum_{i=1}^{n} H\left(i, n, \frac{1}{m}\right) i p_{i}^{*} .
\end{aligned}
$$

The following proposition states the most important results of this section.

Proposition 3. a) If sellers cannot apply state-contingent pricing, then the unique symmetric equilibrium has $p_{i}^{*}=c$ for all $i=\{1, \ldots, n\}$ and $\pi^{*}\left(n, m ; \boldsymbol{p}^{*}\right)=0$.

b) If sellers can apply state-contingent pricing, there exists a continuum of positive profit levels that can be supported in the symmetric equilibrium.

c) In all symmetric equilibria, $U^{*}\left(n, m ; \boldsymbol{p}^{*}\right)>0$.

Proof. a) When sellers cannot price based on ex post realized demand, it must be that $p_{i}^{*}=p^{*}$, for all $i$. Using this fact in (13), together with Facts 1 and 2, immediately implies that $p^{*}=c$. Then, imposing $p^{*}=c$ in (16) yields $\pi^{*}\left(n, m ; \mathbf{p}^{*}\right)=0$.

b) The result is an immediate consequence of the price indeterminacy. Clearly, one can find an infinite combination of prices, $p_{1}^{*}, \ldots, p_{n}^{*}$, such that (13) holds, $p_{i}^{*} \leq u$ for all $i=\{1, \ldots, n\}$, and (14) is satisfied with strict inequality. I just illustrate one: suppose that sellers can choose a price, $p_{1}$, that they will charge if only one customer visits their store, and another price, $p_{2}$, that they will charge if two or more customers show up. Consider the prices $p_{1}=u+(c-u)(m-1)\{[m-F(1, n, m)] H(1, n, m)\}^{-1}$, and $p_{2}=u$, and let $\overline{\mathbf{p}}^{*}=\left(p_{1}, p_{2}, \ldots, p_{2}\right)$. First, notice that the candidate prices satisfy (13). Moreover,

$$
\begin{aligned}
\pi^{*}\left(n, m ; \overline{\mathbf{p}}^{*}\right) & =\frac{n}{m}\left[p_{1} H(1, n, m)+p_{2} \sum_{i=2}^{n} H(i, n, m) i-c\right]= \\
& =(u-c) \frac{n(n-1)}{m\left[(m-1)^{2}+n-1\right]}>0 .
\end{aligned}
$$

Finally, notice that $p_{1}<u$, since, in the definition of $p_{1}$, all the terms multiplying $c-u$ are positive. Thus, I have shown that the candidate prices constitute a symmetric equilibrium associated with positive profit.

c) Suppose, by way of contradiction, that an equilibrium with $U^{*}\left(n, m ; \mathbf{p}^{*}\right)>0$ exists. Since $p_{i}^{*} \leq u$ for all $i,(17)$ indicates that this can only happen if $p_{i}^{*}=u$ for all $i$. However, plugging this fact into (13) implies $u=c$, which is a contradiction.

Proposition 3 indicates that, in the absence of state-contingent pricing, the unique equilibrium satisfies $p^{*}=c$, and the Bertrand paradox emerges. If sellers can post prices based on ex post realized demand, a continuum of equilibria exist, that are not payoff equivalent. To see this point, fix the strategy of all sellers but $j$, and consider this seller's 
best response. When seller $j$ has access to a pricing scheme $p_{i}^{j}$, she can advertise more buyer surplus in some states and less in other states, leaving expected payoffs of other players unaffected. ${ }^{16}$ Intuitively, given any price announcement by her rivals, seller $j$ has a best response correspondence (rather than function), which, by symmetry, gives rise to a continuum of equilibrium prices. More importantly, in the small market, the various best responses are associated with different levels of expected utility in the subgame (since market utility is not fixed), which implies that the sharing rule of the surplus is not uniquely pinned down. The last part of Proposition 3 states that sellers cannot extract the whole surplus in symmetric equilibrium. This would require setting $p_{i}^{*}=u$ for all $i$. However, according to part (a), a constant price necessarily leads to $\pi^{*}\left(n, m ; \mathbf{p}^{*}\right)=0$.

One can describe equilibrium profits in more detail. Replacing the term $c$ from the equilibrium condition (13) into (16), yields

$$
\pi^{*}\left(n, m ; \mathbf{p}^{*}\right)=\frac{n}{m(m-1)} \sum_{i=1}^{n} H\left(i, n, \frac{1}{m}\right) i\left[F\left(i, n, \frac{1}{m}\right)-1\right] p_{i}^{*} .
$$

Clearly, the best equilibrium for the buyers is the Bertrand equilibrium, where $p_{i}^{*}=c$ for all $i$, and $\pi^{*}\left(n, m ; \mathbf{p}^{*}\right)=0$. The best equilibrium for the sellers is achieved under a price scheme $\mathbf{p}^{*}$ that maximizes (18), subject to the constraint that $\mathbf{p}^{*}$ respects (13), and $p_{i}^{*} \leq u$ for all $i$. Typically, the function $G(i) \equiv H(i, n, 1 / m) i[F(i, n, 1 / m)-1]$ is negative for small values of $i$, it becomes positive as $i$ increases, and eventually reaches zero as $i$ gets close to $n$. Hence, an equilibrium $\mathbf{p}^{*}$ associated with high profits tends to assign a greater weight (i.e. a higher value of $p_{i}^{*}$ ) on the terms $G(i)$ that are positive and large, without violating (13) and ex post participation.

As the discussion above reveals, when sellers can advertise prices that differ in each state $i=1, \ldots, n$, the set of possible equilibria is very rich. However, it is important to highlight that all one needs in order to support equilibria with positive profits is some price differentiation among states (i.e. a price schedule that is not fixed). As an example, let $m=2, n=10$, and assume that sellers only have access to the following mechanism: they can choose a price, $p^{-}$, to be charged when five or less buyers show up, and a price, $p^{+}$, to be charged when six or more buyers show up. Then, any $\left(p^{-}, p^{+}\right)$that satisfies

$$
\begin{aligned}
\frac{443}{256} p^{-}-\frac{187}{256} p^{+} & =c, \\
5\left(\frac{1}{2} p^{-}+\frac{1}{2} p^{+}-c\right) & \geq 0,
\end{aligned}
$$

and $p^{-}, p^{+} \leq u$, constitutes a symmetric equilibrium. In Figure 1, the bold red line illustrates the set of all possible equilibrium prices, for $u=1, c=0.2$. Equilibrium profits increase as one moves northwest along this line. The highest equilibrium profit is achieved when $p^{+}=1$ and $p^{-}=0.537$. The best equilibrium for the buyers is represented by the point where the equilibrium line intersects with the 45 degree line, implying that $p^{-}=p^{+}=0.2$ and, hence, $\pi^{*}=0$.

\footnotetext{
${ }^{16}$ This is highlighted by the fact that, although the seller has $n$ control variables, her best response can be summarized by a choice of only one variable: the probability with which the arbitrary buyer visits her store. See, in particular, equation (15).
} 


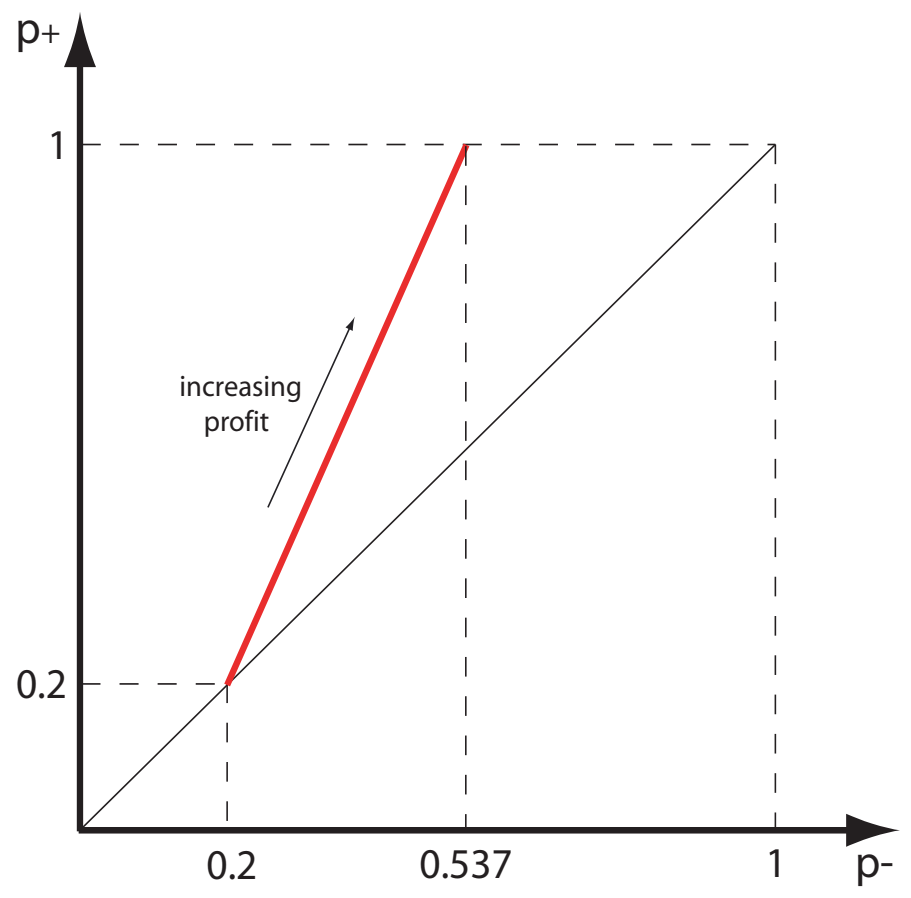

Figure 1: All $p^{-}, p^{+}$on the bold red line are consistent with equilibrium.

\section{Comparison of the Three Environments}

In Sections 3, 4, and 5, I illustrated how the directed search model, augmented with capacity constraints, congestion effects, or state-contingent pricing, respectively, can generate equilibria where the Bertrand paradox does not emerge. In this section, I highlight that these different ingredients lead to the same result (failure of the Bertrand paradox), because they share a common feature. Moreover, I compare equilibrium welfare under the three environments and discuss potential policy implications of my model.

From Section 2, recall equation (2), which I repeat here for convenience,

$$
U^{j}\left(\mathbf{p}^{j}, \theta\right)=\sum_{i=1}^{n} H(i, n, \theta) \min \{i, k\}\left[u(\min \{i, k\})-p_{i}^{j}\right] .
$$

This is the expected utility of a buyer who visits seller $j$, when that seller announces a price schedule $\mathbf{p}^{j}$ and gets visited by an arbitrary buyer with probability $\theta$. If there are no capacity constraints, then $\min \{i, k\}=i$ for all $i \leq n$. If there are no congestion effects, then $u(\min \{i, k\})=u$. Finally, if seller $j$ cannot post state-contingent prices, then $p_{i}^{j}=p^{j}$. In an environment where these three scenarios hold true simultaneously, equation (2) becomes $U^{j}\left(\mathbf{p}^{j}, \theta\right)=u-p^{j}$. In words, buyers get served with certainty at every location, and they pay the announced price. As a result, sellers who announce prices higher than the competition get no customers. This leads to a price war that will end only when all sellers set $p^{*}=c$, so that the Bertrand equilibrium arises. 
It is now clear that the three different ingredients introduced in the model share a common feature. They make a buyer's expected utility, from visiting a certain seller, a function of the total number of visitors at that seller. More precisely, in the presence of any of the three ingredients, buyers tend to dislike sellers with many customers: when $k<n$, many customers could imply rationing; when there are congestion effects, the valuation of the good diminishes in crowded stores; and when sellers can post state-contingent prices, they will tend to charge more when ex post realized demand is high. ${ }^{17}$ In all cases, buyers are willing to visit sellers with higher prices because these sellers will tend to have fewer customers. Sellers accept the offer and indeed charge higher prices in equilibrium. To use the game theory jargon, the existence of (any of) the three ingredients studied in this paper, serves as a collusion device that allows sellers to achieve positive profits.

I believe that this finding is of great importance. The IO literature has emphasized the existence of capacity constraints as the most prominent resolution to the Bertrand paradox, at least in markets with homogeneous good and sellers. This paper highlights that the existence of capacity constraints is just a subcase of a more general market description in which the Bertrand paradox fails to hold: equilibrium profits will be positive in markets where the buyers' expected utility from visiting a seller is a (decreasing) function of that seller's ex post realized demand. This negative relationship (between expected utility and the number of customers) will be satisfied if sellers face capacity constraints, but it will also be satisfied if there are congestion effects and/or if sellers have access to state-contingent pricing. It should be pointed out that the choice to study oligopolistic competition in a directed search model is crucial in order to arrive at this generalization.

I now examine the welfare properties of equilibria under the three environments. Define the expected total surplus, $\mathcal{S}^{*} \equiv n U^{*}+m \pi^{*}$, where $\pi^{*}$ is expected profit per seller, and $U^{*}$ is expected net utility per buyer, in the symmetric equilibrium. One can show that in the model with capacity constraints, congestion effects, and state-contingent pricing, respectively, the expected total surplus is given by

$$
\begin{aligned}
\mathcal{S}^{*}(n, m ; k) & =n(u-c) \sum_{i=1}^{n} H\left(i, n, \frac{1}{m}\right) \min \{i, k\}, \\
\mathcal{S}^{*}(n, m ; u) & =n \sum_{i=1}^{n} H\left(i, n, \frac{1}{m}\right) i[u(i)-c], \\
\mathcal{S}^{*}\left(n, m ; \mathbf{p}^{*}\right) & =n(u-c) .
\end{aligned}
$$

In the model with capacity constraints, every match generates a surplus equal to $u-c$, but matches are not guaranteed. Since $\sum_{i=1}^{n} H(i, n, 1 / m) \min \{i, k\}$ is the probability with which a buyer gets served in the symmetric equilibrium, multiplying that term with $n$ yields the total number of expected matches in the economy (or the matching function). Clearly, $\mathcal{S}^{*}(n, m ; k)$ is strictly increasing in $k$, for all $k \leq n$. In the model with congestion

\footnotetext{
${ }^{17}$ To be more exact, in this paper I document the existence of multiple equilibria, but I do not claim that equilibria with higher profits will emerge with higher probability. This statement just draws on the discussion of Section 5 , where I showed that higher equilibrium profits are linked to higher prices in states where ex post realized demand is high.
} 
effects, all buyers get served. A buyer finds herself at a store with a total of $i$ customers with probability $H(i, n, 1 / m) i$. In this event, a surplus $u(i)-c$ per person is generated. Stronger congestion effects imply a lower value of $\mathcal{S}^{*}(n, m ; u)$. Finally, in the model with state-contingent pricing, all buyers get served, and their valuation of the good does not depend on the number of visiting customers (although the price they pay does).

Most modern economies enforce antitrust laws whose main objective is to prevent anticompetitive practices, most notably price fixing. ${ }^{18}$ This paper highlights that sellers can boost profits above the Bertrand level, without directly fixing prices. More importantly, the practices that help sellers sustain prices above marginal cost, have different consequences on social welfare. For example, state-contingent pricing is a practice that allows sellers achieve positive equilibrium profits, but does not affect total welfare. Therefore, in this environment, the authorities should intervene only if they judge that the sharing rule of the surplus is unfair, e.g. sellers are making excessive profits.

Now consider the model with capacity constraints. The total surplus, $\mathcal{S}^{*}$, is strictly increasing in $k$. However, the discussion in Section 3 reveals that there exists a unique $\iota<n$, such that $\pi^{*}(n, m ; k)$ is decreasing in $k$, for all $k \geq \iota$. Hence, if sellers can fix $c a-$ pacities, typically, they will not choose the socially optimal $k$. As an example, consider a market with $m=5, n=50, u=1$, and $c=0.2$. Suppose that sellers can silently agree on a value of $k$, and then, given that $k$, they legitimately compete over prices. Sellers would set $k=7$ since, under this value, the per seller equilibrium profit is maximized and equals $\pi^{*}(50,5 ; 7)=4.692$. At the same time, $\mathcal{S}^{*}(50,5 ; 7)=27.29$. If the authorities could enforce an increase of $k$ by just one unit, the surplus would increase to $\mathcal{S}^{*}(50,5 ; 8)=30.53$, and this would lead to $\pi^{*}(50,5 ; 8)=4.690$. If $k=9$, then $\mathcal{S}^{*}(50,5 ; 9)=33.30$, and if $k=10$, then $\mathcal{S}^{*}(50,5 ; 10)=35.525 .^{19}$

Finally, consider the policy implications for the model with congestion effects. For concreteness, consider the numerical example introduced in Section 4, i.e. assume that $u(i)=v-\kappa(i-1)$, with $\kappa>0, v>c$, and $\kappa \leq(v-c) /(2 n-1)$. Under this specification, the expected total surplus becomes

$$
\mathcal{S}^{*}(n, m ; u)=n \sum_{i=1}^{n} H\left(i, n, \frac{1}{m}\right) i[v-\kappa(i-1)-c]=\frac{n[m(v-c)-\kappa(n-1)]}{m} .
$$

This expression is decreasing in the size of the congestion effect, $\kappa$. However, recall from the discussion in Section 4, that $\pi^{*}=\kappa[n(n-1)][m(m-1)]^{-1}$, which is increasing in $\kappa$. Hence, in this market, there is a clear conflict: sellers are better off when $\kappa$ is large, but social welfare is maximized when $\kappa=0$. The authorities should be concerned about this conflict, since sellers have a clear incentive to agree on practices that increase $\kappa$ and, thus, decrease $\mathcal{S}^{*}$ artificially (and -importantly- do not violate any antitrust laws). Such practices could include allowing big queues at the stores or hiring too few employees who cannot provide quality services when many customers show up.

\footnotetext{
${ }^{18}$ Other practices that antitrust laws aim to prevent include formation of cartels, barriers to entry, bid rigging, product bundling, exclusive dealing, and misuse of patents and copyrights.

${ }^{19}$ For these values of $k$ the equilibrium profits become $\pi^{*}(50,5 ; 9)=4.352$ and $\pi^{*}(50,5 ; 10)=3.727$. Also, notice that $k$ does not need to be too close to $n$ for $\mathcal{S}^{*}$ to reach its upper bound, $n(u-c)$. Here $n=50$, but for a $k$ as low as 13 , one obtains $\mathcal{S}^{*}(50,5 ; 13)=39.1$, which is very close to $n(u-c)=40$.
} 


\section{Conclusions}

In this paper, I revisit the Bertrand Paradox through the lens of a directed search model. I augment the baseline model with three ingredients, capacity constraints, consumption externalities, and state-contingent pricing, in isolation, and I show that in each case the Bertrand Paradox does not arise. The three different ingredients share a common feature: they make a buyer's expected utility from visiting a specific seller, a decreasing function of that seller's ex post realized demand. In all three cases, buyers dislike stores with many customers and, thus, are willing to visit sellers with higher prices. This economic force leads to equilibria where sellers indeed post prices higher than the marginal cost. The directed search model offers a new perspective of looking at the Bertrand Paradox, by highlighting that the existence of capacity constraints is just a subcase of a more general class of environments where the paradox fails to hold.

\section{A Appendix}

Proof of the fact that $p^{*}(n, m ; k)$ is strictly decreasing in $k$. For the economy of space, I write $H(i)$ for $H(i, n, 1 / m)$ and $F(i)$ for $F(i, n, 1 / m)$. I show that for all $k=1, \ldots, n-1$, $D p^{*}(k) \equiv p^{*}(n, m ; k)-p^{*}(n, m ; k+1)>0$. To start, add and subtract the term $m(m-$ $1)^{-1} c \sum_{i=1}^{n} H(i) \min \{i, k\}[1-F(i)]$ in the numerator of $p^{*}$ in (7). This allows one to re-write the equilibrium price as

$$
p^{*}(n, m ; k)=c+m(u-c) \frac{\sum_{i=1}^{n} H(i) \min \{i, k\}[1-F(i)]}{\sum_{i=1}^{n} H(i) \min \{i, k\}[m-F(i)]} .
$$

Using (a.1), for any $k<n-1$,

$$
D p^{*}(k)=\frac{m(u-c) \Omega}{\sum_{i=1}^{n} H(i) \min \{i, k\}[m-F(i)] \sum_{i=1}^{n} H(i) \min \{i, k+1\}[m-F(i)]},
$$

where I have defined the term

$$
\begin{aligned}
\Omega & \equiv \sum_{i=1}^{n} H(i) \min \{i, k\}[1-F(i)] \sum_{i=1}^{n} H(i) \min \{i, k+1\}[m-F(i)] \\
& -\sum_{i=1}^{n} H(i) \min \{i, k+1\}[1-F(i)] \sum_{i=1}^{n} H(i) \min \{i, k\}[m-F(i)] .
\end{aligned}
$$

The proof of monotonicity of $p^{*}$ will be complete, if I can show that the denominator of the expression on the right-hand side of (a.2) and the term $\Omega$ share the same sign. I claim that they are both positive, and I prove this claim below.

Claim 1: Both summations in the denominator of (a.2) are positive. I show the result in detail for the first term. The proof is identical for the second term. Since $m \geq 2$, and $H(i)>0$ for all $i=1, \ldots, n$, it is true that $\sum_{i=1}^{n} H(i) \min \{i, k\}[m-F(i)]>$ 
$\sum_{i=1}^{n} H(i) \min \{i, k\}[1-F(i)]$. I will now show that $\sum_{i=1}^{n} H(i) \min \{i, k\}[1-F(i)]>0$.

It follows from Facts 1,2 that $\sum_{i=1}^{n} H(i) i[1-F(i)]=0$. Moreover, the function $1-F(i)$ is strictly decreasing in $i$, for all $i \leq n$, and satisfies $1-F(1)=(n-1) /(m-1)>0$, and $1-F(n)=1-n<0$. Hence, there exists a unique $\nu \in\{1, \ldots, n-1\}$, such that $H(i) i[1-F(i)] \geq 0$ iff $i \leq \nu$, and one can write

$$
\sum_{i=1}^{\nu} H(i) i[1-F(i)]=-\sum_{i=\nu+1}^{n} H(i) i[1-F(i)] .
$$

Assume, without loss of generality, that $k \geq \nu$ and notice that all terms on the right-hand side of (a.3) are positive. This allows me to write

$$
\begin{gathered}
\sum_{i=1}^{\nu} H(i) i[1-F(i)]>-\sum_{i=\nu+1}^{k} H(i) i[1-F(i)]-k \sum_{i=k+1}^{n} H(i)[1-F(i)] \Leftrightarrow \\
\sum_{i=1}^{n} H(i)[1-F(i)] \min \{i, k\}>0 .
\end{gathered}
$$

This concludes the proof of the first claim. ${ }^{20}$

Claim 2: $\Omega$ is positive. After a series of manipulations, one can obtain

$$
\Omega=(m-1)\left[\sum_{i=1}^{k} H(i) i \sum_{i=k+1}^{n} H(i) F(i)-\sum_{i=1}^{k} H(i) F(i) i \sum_{i=k+1}^{n} H(i)\right],
$$

and it suffices to show that

$$
\sum_{i=1}^{k} H(i) i \sum_{i=k+1}^{n} H(i) F(i)>\sum_{i=1}^{k} H(i) F(i) i \sum_{i=k+1}^{n} H(i) .
$$

After multiplying throughout with $i$, the last inequality becomes

$$
\sum_{i=1}^{k} H(i) i \sum_{i=k+1}^{n} H(i) F(i) i>\sum_{i=1}^{k} H(i) F(i) i \sum_{i=k+1}^{n} H(i) i .
$$

Finally, use Facts 1 and 2 to replace $\sum_{i=k+1}^{n} H(i) F(i) i$ and $\sum_{i=k+1}^{n} H(i) i$ in (a.4). My original claim, that $\Omega>0$, will hold if and only if

$$
\sum_{i=1}^{k} H(i) i[1-F(i)]>0
$$

This inequality holds for every parameter value, since $\sum_{i=1}^{n} H(i) i[1-F(i)]=0$, and the function $1-F(i)$ is strictly decreasing in $i$. Hence, the proof is complete.

${ }^{20}$ The intuition of this proof can be summarized as follows. Since the summation (for $i=1$ to $n$ ) of the terms $H(i) i[1-F(i)]$ adds up to zero, and the terms associated with larger values of $i$ are negative, the expression $\sum_{i=1}^{n} H(i)[1-F(i)] \min \{i, k\}$ tends to assign a smaller "weight" on the negative terms, thus adding up to a value bigger than zero. 


\section{References}

Acemoglu, D., And R. Shimer (1999): "Efficient Unemployment Insurance," Journal of Political Economy, 107(5), 893-928.

Burdett, K., S. Shi, And R. Wright (2001): "Pricing and Matching with Frictions," Journal of Political Economy, 109(5), 1060-1085.

Coles, M. G., and J. Eeckhout (2003): "Indeterminacy and directed search," Journal of Economic Theory, 111(2), 265-276.

Cournot, A. (1838): Recherches sur les principes mathématiques de la théorie des richesses/par Augustin Cournot. L. Hachette.

Dudey, M. (1992): "Dynamic Edgeworth-Bertrand competition," The Quarterly Journal of Economics, 107(4), 1461-1477.

Edgeworth, F. (1897): "La teoria pura del monopolio," Giornale degli economisti, 40, 13-31.

Galenianos, M., and P. Kircher (2009): "Directed search with multiple job applications," Journal of Economic Theory, 144(2), 445-471.

Geromichalos, A. (2009): "Directed search and optimal production," .

JACQUET, N., AND S. TAN (2012): "Wage-vacancy contracts and coordination frictions," Journal of Economic Theory.

Julien, B., J. Kennes, And I. King (2000): "Bidding for labor," Review of Economic Dynamics, 3(4), 619-649.

Kreps, D., And J. Scheinkman (1983): "Quantity precommitment and Bertrand competition yield Cournot outcomes," The Bell Journal of Economics, pp. 326-337.

LAgos, R. (2000): "An Alternative Approach to Search Frictions," Journal of Political Economy, 108(5), 851-873.

LANG, K. (1991): "Persistent wage dispersion and involuntary unemployment," The Quarterly Journal of Economics, 106(1), 181-202.

LESTER, B. (2010): "Directed search with multi-vacancy firms," Journal of Economic Theory.

(2011): "Information and prices with capacity constraints," The American Economic Review, 101(4), 1591-1600.

Montgomery, J. D. (1991): "Equilibrium Wage Dispersion and Interindustry Wage Differentials," The Quarterly Journal of Economics, 106(1), 163-79. 
Peters, M. (1984): "Bertrand equilibrium with capacity constraints and restricted mobility," Econometrica: Journal of the Econometric Society, pp. 1117-1127.

Shaked, A., And J. Sutton (1982): "Relaxing price competition through product differentiation," The Review of Economic Studies, pp. 3-13.

TAN, S. (2010): "Directed Search and Firm Size," National University of Singapore mimeo.

VIRAG, G. (2007): "Collusive equilibria in directed search models," University of Rochester mimeo.

Watanabe, M. (2010): "Middlemen: A Directed Search Equilibrium Approach," Universidad Carlos III de Madrid mimeo. 